\title{
Improvement in a Patient with Active Systemic Lupus Erythematosous Treated with Transplant of Intestinal Microbiota
}

ISSN: 2637-7659

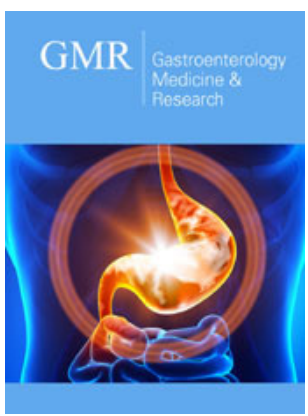

For HTML Version scan this QR code:

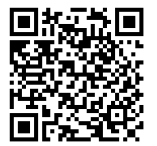

*Corresponding author: Álvaro Zamudio Tiburcio, Department of Gastroenterology, México

Submission: February 15, 2019

Published: :-iln March 01, 2019

Volume 3 - Issue 1

How to cite this article: Álvaro Z-T Héctor B-R, Pedro A R-L. Improvement in a Patient with Active Systemic Lupus Erythematosous Treated with Transplant of Intestinal Microbiota. Gastro Med Res.3(1). MCDA.000551.2019.

DOI: 10.31031/GMR.2019.03.000551

Copyright@ Álvaro Zamudio Tiburcio, This article is distributed under the terms of the Creative Commons Attribution 4.0 International License, which permits unrestricted use and redistribution provided that the original author and source are credited.

\section{Álvaro Zamudio-Tiburcio ${ }^{1 *}$, Héctor Bermúdez-Ruiz ${ }^{2}$ and Pedro Antonio Reyes- López ${ }^{3}$}

${ }^{1}$ Department of Gastroenterology, México

${ }^{2}$ Department of Endoscopy, México

${ }^{3}$ Department of Cardiology, México

\begin{abstract}
We analyzed a single case of a patient with active Systemic Lupus Erythematosus (SLE), transplanted with Intestinal Microbiota (IMT). She had 34 years old, with active SLE with 9 years of evolution. She has lupic glomerulonephritis and the last 6 months diarrhea, the she loss weight $(28 \mathrm{~kg})$ and had desnutrition (BCI 16). Treated with racecadotrilo and loperamide. A coprologic study showed Blastocystis hominis. She did not received treatment for this parasite. An IMT was done 9 months ago, reducing diarrhea and anxiety. After a week she was hospitalized because diarrhea, deshydratation. Under care of a nephrologist who prescribe Mycophenolic acid, and nothing change. A different nephrologist stops Mycophenolic acid and prescribe Azathioprine resulting in improvement when nausea and diarrhea ceased. Next day she was eating and recovered $7 \mathrm{~kg}$, however, she was anemic (her hemoglobin was $8 \mathrm{~g} / \mathrm{dL}$ ) and she was transfused with total blood. Weights 15 more kilograms, oscillating between 52 to $55 \mathrm{~kg}$ and her was asymptomatic. $55 \mathrm{k}$. She offered testimony of her improvement. https://goo.gl/oHZeUT
\end{abstract}

Keywords: Intestinal microbiota transplantation; Systemic lupus erythematosous, Lupus and microbiota

\section{Introduction}

After the good response to treatment in a case of Clostridium difficile IMT [1] other gastrointestinal diseases, such as Irritable Bowel Syndrome [2-4] other intestinal inflammatory condition such as UC and Crohn, showed good results [5-8], other chronic conditions such as neurologic, dermatologic, and autoimmune conditions treated with IMT had good responses, in Multiple sclerosis [9] Parkinson [10] metabolic syndrome [11], Irritable bowel syndrome (IBS) [12] chronic constipation [13] Thrombocytopenic purpura [14] and refractory "Pouchitis" [15,16].

\section{Clinical Case}

A female of 34 y.o. who had a loss weight of $28 \mathrm{~kg}$ since the last year, with diarrhea, disseminated arthralgias, anxiety, depression, headaches, dysuria, abdominal distention, cholic pains, leg edema, eructs, fetid gases expulsed, halitosis, nauseas, polydipsia, gastreoesophageal reflux, somnolency and occasional vomits. With normal urine, clinical chemistry, and minimum changes in her hematic biometry; presence of untreated Blastocystis hominis; and normal colonoscopy; has abnormal immunologic studies.

Her hemolytic Complement activity 50\% was 99.56 units CEA.

Reference: 63 -145 normal CEA units.

Complement C3 fraction: 67.9mg/dl. Normal reference $90-180 \mathrm{mg} / \mathrm{dl}$

Complement C4 fraction: $17.4 \mathrm{mg} / \mathrm{dl}$. Normal reference $10-40 \mathrm{mg} / \mathrm{dl}$

Antinuclear Antibodies positive, dilution: 1:160*.

Reference: Negative. 
Ac anti-SSA: 4.0. $\mathrm{U}^{*}$

Reference: $<0.84$ Ratio

Ac anti-SSB: $2.6 \mathrm{U} / \mathrm{mL}$

Reference: $<15 \mathrm{U} / \mathrm{mL}$

Ac anti-SM: $28.8 \mathrm{U} / \mathrm{mL}^{*}$

Reference: $<15 \mathrm{U} / \mathrm{mL}$

In addition, she has allergy to chocolate, had ureteral dilatation. Never was pregnant, has systemic hypertension, and smoked 10 cigarettes/day between 17 to 29 years old.

Her diagnostics are:

Systemic Lupus Erythematosus, active, since the last 9 years.

Lupus glomerulonephritis for 9 months.

Chronic Diarrhea for 1 year. 1chronic since 1year.

Denutrition for 7 months (BCI: 16).

Anxiety.

Skin: was tattooed.

\section{Medical Treatment}

Mycophenolic acid, comprimides (500mg) 1 x 3. Pentoxifylline, dragees (400mg) 1 x 1 . Pioglitazone, Tablets (15mg) 1 x 1 . Bumetanide, Tablets (1mg) 1 x 1 . Deflazacort, Tablets (6mg) $1 / 2$, daily. Metoxi-polietilenglicol epoetina beta, $(200 \mathrm{mcg} 0.3 \mathrm{ml}$ injectable solution, precharged syringe) Monthly. SC. Febuxostat, Tablets (80mg) $1 \times 1$., comprimids (600mg) $1 \times 1$. Ezetimibe and simvastatin, comprimids (10/20) $1 \times 1$. Losartan, tablets, (50mg) 1 x 2. Pantoprazole, tablets ( $40 \mathrm{mg}) 1 \times 1$. Aminoacid-alfacetoanalogs, comprimids, 6 daily. Racecadotrile, capsuls (100mg) 1 x 1 . Nifuroxazida, Cápsules (400mg) 1 x 2; severe diarrhea: Neomycin + kaolin + pectina, Tablets. $1 \times 3$.

Bacillus Clausii, ampoules (5ml) 3 ampoules /day. Loperamide Clorhydrate Tablets (2mg) 1 x 1 . Bifidobacterium Lactis; carbohydrates; greases, Lactobacillus Acidophilus; Lactobacillus Casei, variety rhamnosus; minerals; proteins; vitamins. 1 x 1 . Diosmectite, envelopes (3G) occasionally. Pancreatin. Dimeticone, dragees. 1 × 3 . Amylase + lipase + protease, capsules $(300 \mathrm{mg}) 1 \times 3$. Alverine-simeticone, capsules $(60 / 300 \mathrm{mg}) 1 \times 3$. Norphenylephrine Clorhydrate, drops. In case of severe arterial hypertension.

Height 1.70; pulse 89X'; breaths 18x'; A.T. 120/75; Weight 46k.; BCI: 16.

She had intestinal microbiota transplant (IMT) through colonoscopy, leaving 500g. In ascending colon 200g, in transverse colon, another $100 \mathrm{~g}$ and $200 \mathrm{~g}$ more in descending colon. Incidents: none. Post transplant evolution was good, her chronic diarrhea ceased. She received Racecadotril, capsules $(100 \mathrm{mg}$ ) and Loperamyde clorhydrate tablets ( $2 \mathrm{mg}$ ) ( 1 x 1 , until liquid diarrhea stoped). She was asymptomatic for a week. However, she was rehospitalized because a transient diarrhea to prevent dehydration.

\section{Discussion}

Systemic Lupus Erithematosous (SLE), is a complex autoimmune disease because the normal tolerance to our own tissues is lost, therefore an inflammatory condition exists, nuclear antigens react with abnormal antibodies forming immune complexes and these immune complexes (IC) deposits on many organs and inflammation mediated by IC deposition cause dysfunction in many important organs. Although there are many studies the intímate cause of autoimmunity remains poorly understood. At present it is known that certain genes are associated with this disease, but autoimmunity is the cause of SLE. Also, it has been considered a possible that autoimmunity was present in SLE and other autoimmune diseases, could be associated to changes in intestinal microbiota.

There are new knowledge suggesting that symbiotic bacteria could modulates the SLE and autoimmunity in general. It is possible that intestinal microbiota could be an important role as environmental factor in human health. Not only in autoimmunity, the concept has been extended to other metabolic, inflammatory, and other chronic diseases. The interaction between intestinal microbiota and unknown genes could be an important factor to etiopathogenesis of many diseases with perturbed responses to intestinal microbiota, the present case suggest the important role of intestinal microbiota interacted with genes to be part of a complex disease, such metabolic, neurologic, immunologic diseases and although it is not possible to demonstrate a cause, the evidence of changes in the interaction of microbiota and unknown human genes could be responsible of a variety of human health problems [17].

Hevia \& cols [18] realized a transversal study to determine if dysbiosis intestinal is associated to SLE. They study SLE without active disease in clinical terms to determine if intestinal dysbiosis intestinal is associated to SLE in patients without active SLE. They studied 20 patients with inactive SLE, with strict criteria for inclusion/exclusion using an optimized protocol of Ion Torrent 16S rRNA to descifrated fecal microbiota pattern in these 20 patients, and compared with 20 normal subjects, healthy, with the same SLE subjects to look after fecal profiles of both groups. They discover that diversity is comparable using the Shannon index (measures specific biodiversity). They found a relationship between Firmicutes/Bacteroides significative low in people with SLE (mean proportion 1.97) in healthy subjects (median relation of 4.86; $\mathrm{p}<0,002$ ). This dysbiosis was reflecting, in base to functional inference in silico, overrepresenting oxidative phosphorylation and the utilization of glycans in the microbiota of SLE patients.

Other authors [19] search the effects of genetic in the host, sex, age and intervention and dietetic in the intestinal microbiome in a murine lupus model. In young females propensity to SLE was similar to the same fertile women. They found increased risk for lupus and less presence of lactobacilli with higher increase in Lachnospiraceae and a general diversity, comparing with general diversity, and in comparison, with healthy controls with the same age. The predicted metagenomic profile for mouse propense to SLE 
was diverse in general, comparing with healthy control of same age. The predicted metagenomic profile in mice propended to lupus shown a significative enrichment of healthy controls of same age. The predicted metagenomic profile in mice propense to SLE shows a significative enrichment of ways related to bacterial motility and sporulation. The retinoic acid restored population predicted in mouse with lupus propensity shown significative enrichment in the way related to bacterial motility with sporulation.

Retinoic acid restored lactobacillis who negatively in mice propense to lupus and this correlated with improved symptoms. The intestinal microbiota of mice propense to lupus was different among sex, an shows an overrepresented Lachnospiraceae in females with early initiation was different among sex and had an overrepresentation of Lachnospiraceae in mice propense to lupus was different between sexes females has early initiation and more severe symptoms. The clostridiáceas and lacnospiraceae, hosted subject who produce butyrate, were more abundant in the gut of mice with lupus propensity who host genders who synthetize butyrate, these were more abundant in the gut or lupus propense, in specific moments and progression. Their results shown a dynamic of intestinal microbiota in murine lupus and give evidence to suggest lactobacilli and retinoic acid improves inflammation in lupus patients.

The principal function of microbiota is the intestine protection against colonization for exogenous pathogens and indigenous microorganisms potentially damaging through several mechanisms, including direct competence for limited nutrients and modulation of host immune response. Instead, pathogens developed strategies to promote their replication in presence of competitive microbiota. The collapse of normal bacterial community increases risks of infection by pathogens, excessive growing of pathobionts and inflammatory diseases. To comprehend interaction between microbiota and pathogens and the host could be with new knowledge on the pathogenesis of disease, to prevention and treatment intestinal, and systemic problems [20].

In the last years, there are studies informing over incidence of SLE in the United States of America. Some studies said that it was an increment by 3 . It was formulated and hypothesis of augmenting incidence of autoimmune diseases due to considerable changes in gut resident bacteria's (intestinal microbiota), after considerable changes in the resident bacterial communities (intestinal microbiota), following changes in diet an indiscriminate antimicrobial treatment $[21,22]$.

López P [23] and collaborators said that the microbiota isolate from feces of SLE (SLE-M) promotes activation of lymphocytes to differentiation Th 17 lymphocytes CD4+ preferentially of normal healthy controls. Enrichment of SLE-M with bacteria's inductors of Treg shown que the admixture of strains of Clostridia reduced significatively the equilibrium Th17 / Th1, instead the supplementing with Bifidobacterium bifidum prevents sobreactivation of $\mathrm{CD}^{+}$, supporting a possible therapeutic benefit of probiotics strains Treg-inductor to participate in restoring the disequilibrium Treg / Th17 / Th1 present in SLE. All of these manifestations of the ingerency of microbiota, above all intestinal microbiota in modulation of lupus disease. That is the reason to direct our bacteria against this cause and inside, with the final to determine this new etiology promotes disease. There are studies mentioning the necessity of probiotics to have a principal role of disease and determines intestinal dysbiosis, characterized by the relationship Firmicutes/Bacteroidetes reduced, in SLE [24-27].

The microbiota, and his effect on the human host is now and major interest for humans. It was demonstrated several parameters of health and disease associated with variations of intestinal microbiome. In recent years, many studies demonstrate the role of intestinal microbiota, not only the classic autoimmune, there are now: diabetes mellitus type 1 , rheumatoid arthritis and multiple sclerosis [28]. Despite the disease mechanism involves genetic and environmental factors, have been discovered in lupus the affection by gut microbiota composition. Recently several studies suggested alterations in the microbial intestine could be correlated with disease manifestations. At the time the exact functions of symbiotic microbial or pathogenic population [29].

When the exact role of intestinal role demonstrates the role of gut microbiota population, our knowledge of pathogenesis of SLE will be, hopefully, oriented how SLE developed and hopefully this knowledge allows better treatments, maybe we will have to offer best opportunities to developed treatments using biomarkers of SLE to offer better treatment and new therapies. This knowledge allows us to better understand intestinal microbiome, and through diet modification and other therapeutic measures we can modify the intestinal microbiome [30,31]. The only cytochime with participation in pathogenesis of SLE is the interferon alpha. Its secretion is induced by immune complexes had a role in this immune complexes disease, through positive regulation of several proinflammatory proteins, this is the current explanation, denominated "signature" of IFN found in many PBMC in SLE. Also, IL-6, IFN and several T cell cytokines such as as IL-17, IL-21and IL-2 are dysregulated in SLE and these induced a phenotype change in $\mathrm{T}$ cells characterized for help to $\mathrm{B}$ cells and potent secretion of B cells, with proinflammatory cytoquins, and reduced induction of supressors T cells and cells death by these active cells. This document will have focus on these cytokins and signall the cytokins results in the physiopathology mechanism of SLE and the therapeutic potential [32]. All of these will be support to explorer the IMT, which is the case in this paper.

\section{Conclusion}

Although SLE has been attributed to many causes, such as subgingival microbiota, which is a complication of this local microbiota. Are many times more a consequence of dysbiosis in the intestinal microbiota? However, all evidences will be respected and studied as explanation, perhaps a partial on this disease [33-35]. The intestinal dysbiosis, characterized by a reduction of Firmicutis / Bacteroidetes ratio, has been notified in patients with SLE [36]. The dysbiosis of intestinal microbiota have been informed in several autoimmune diseases, however, the role of intestinal microbiota in SLE is relatively new. This autoimmune prototypic disease, 
characterized by persistent inflammation in multiple organs, still is difficult of explain. We considered that IMT will be explored in deep in this disease.

\section{References}

1. Russell GH, Kaplan JL, Youngster I, Baril-Dore M, Schindelar L, et al. (2014) Fecal transplant for recurrent Clostridium difficile infection in children with and without inflammatory bowel disease. J Pediatr Gastroenterol Nutr 58(5): 588-592.

2. Mayer EA, Savidge, Shulman RJ (2014) Brain-gut microbiome interactions and functional bowel disorders. Gastroenterology 146(6): 15001512.

3. Borody TJ, George L, Andrews P, Brandl S, Noonan S, et al. (1989) Bowel-flora alteration: A potential cure for inflammatory bowel disease and irritable bowel syndrome? Med J Aust 150(10): 604.

4. Allegretti JR, Hamilton MJ (2014) Restoring the gut microbiome for the treatment of inflammatory bowel diseases. World J Gastroenterol 20(13): 3468-3474.

5. Aroniadis OC, Brandt LJ (2013) Fecal microbiota transplantation: Past, present and future. Curr Opin Gastroenterol 29(1): 79-84.

6. Sha S, Liang J, Chen M, Xu B, Liang C, et al. (2014) Systematic review: Faecal microbiota transplantation therapy for digestive and Non-digestive disorders in adults and children. Aliment Pharmacol and Therapeutics 39(10): 1003-1032.

7. Borody TJ, Warren EF, Leis Surace R, Ashman O (2003) Treatment of ulcerative colitis using fecal bacteriotherapy. J Clin Gastroenterol 37(1): 42-47.

8. Halkjær SI, Boolsen AW, Günther S, Christensen AH, Petersen AM (2017) Can fecal microbiota transplantation cure irritable bowel syndrome? World Gastroenterolog 23(22): 4112-4120.

9. Borody TJ, Leis SM, Campbell J, Torres M, Nowak A (2013) Fecal microbiota transplantation (FMT) in multiple sclerosis (MS). Am J Gastroenterol 15: 337.

10. Ananthaswamy A (2011) Faecal transplant eases symptoms of Parkinson's. New Scientist.

11. Vrieze A, van Nood E, Holleman F, Salojarvi J, Kootte RS, et al. (2012) Transfer of intestinal microbiota from lean donor's increases insulin sensitivity in individuals with metabolic syndrome. Gastroenterology 143(4): 913-967.

12. Simrén M, Barbara G, Flint HJ, Spiegel BM, Spiller RC, et al. (2013) Intestinal microbiota in functional bowel disorders: a Rome foundation report. Gut 62(1): 159-176.

13. Borody TJ, Leis S, McGrath K, Spence E, Surance R, et al. (2001) Treatment of chronic constipation and colitis using human probiotic infusions. Probiotics, Prebiotics and New Foods Conference, Universita Urbaniana, Rome, Italy.

14. Borody TJ, Torres M, Nowak A, Leis S (2011) Reversal of idiopathic thrombocytopenic purpura with fecal microbiota transplantation [FMT]. Am J Gastroenterol 106: S352.

15. Zhang FM, Wang HG, Wang M, Cui BT, Fan ZN, et al. (2013) Fecal microbiota transplantation for severe entero-colonic fistulizing Crohn's disease. World J Gastroenterol 19(41): 7213-7216.

16. Landy J, Omar H, Mann E, S T Peake, S D McLaughlin, et al. (2013) A prospective controlled pilot study of faecal microbiota transplantation for chronic refractory pouchitis. Journal of Crohn's \& Colitis 7: S247-S248.
17. Ahern PP, Faith JJ, Gordon JI (2014) Mining the human gut microbiota for effector strains that shape the immune system. Immunity 40(6): 815823.

18. Hevia A, Milani C, López P, Cuervo A, Arboleya S, et al. (2014) Intestinal dysbiosis associated with systemic lupus erythematosus. MBio 5(5): e01548-14.

19. Zhang H, Liao X, Sparks JB, Luo XM (2014) Dynamics of gut microbiota in autoimmune lupus. Appl Environ Microbiol 80(24): 7551-7560.

20. Kamada N, Seo SU, Chen GY, Nuñez G (2013) Role of the gut microbiota in immunity and inflammatory disease. Nat Rev Immunol. 13(5): 321-335.

21. Rosser EC, Mauri C (2016) A clinical update on the significance of the gut microbiota in systemic autoimmunity. J Autoimmun 74: 85-93.

22. Neuman H, Koren $\mathrm{O}$ (2017) The gut microbiota: a possible factor influencing systemic lupus erythematosus. Curr Opin Rheumatol 29(4): 374377.

23. López P, de Paz B, Rodríguez-Carrio J, Hevia A, Sánchez B, et al. (2016) Th17 responses and natural IgM antibodies are related to gut microbiota composition in systemic lupus erythematosus patients. Scientific Report 6: 24072 .

24. Luo XM, Edwards MR, Mu Q Yu Y, Vieson MD, et al. (2018) Gut Microbiota in Human Systemic Lupus Erythematosus and a Mouse Model of Lupus. Applied and Environmental Microbiology 84(4): 1-10.

25. Gul'neva M, Romanov VA, Shilkina NP (2007) Intestinal microecology in some systemic connective tissue diseases. Zh Mikrobiol Epidemiol Immunobiol 4: 38-41.

26. Mu Q, Zhang H, Liao X, Lin K, Liu H, et al. (2017) Control of lupus nephritis by changes of gut microbiota. Microbiome 5: 73 .

27. Katz-Agranov N, Zandman-Goddard G (2017) The microbiome and systemic lupus erythematousus. Immunol Res. 65(2): 432-437.

28. Palm NW, de Zoete MR, Flavell RA (2015) Immune-microbiota interactions in Health and disease. Clin Immunol 159: 122-127.

29. Khanna S, Tosh PK (2014) A clinician's primer on the role of the microbiome in human health and disease. Mayo Clin Proc 89(1): 107-114.

30. Kosiewicz MM, Zirnheld AL, Alard P (2011) Gut microbiota, immunity, and disease: a complex relationship. Front Microbiol 2: 180.

31. Ohl K, Tenbrock K (2011) Inflammatory cytokines in systemic lupus erythematosus. J Biomed Biotechnol 2001: 432595.

32. Gao L, Xu T, Huang G, Jiang S, Gu Y, et al. (2018) Oral microbiomes: more and more importance in oral cavity and whole body. Protein Cell 9(5): 488-500.

33. Dias Corrêa J, Cerqueira Calderaro D, Aparecida Ferreira G, Souza Mendoça SM, Fernandes GR, et al. (2017) Subgingival microbiota dysbiosis in systemic lupus erythematosus: association with periodontal status. Microbiome 5: 34 .

34. Fabbri C, Fuller R, Bonfá E, Guedes LKN, D’Alleva PSR, et al. (2014) Periodontitis treatment improves systemic lupus erythematosus response to immunosuppressive therapy. Clin Rheumatol 33(4): 505-509.

35. de Araújo Navas EAF, Sato EI, Pereira DFA, Back-Brito GN, et al. (2012) Oral microbial colonization in patients with systemic lupus erythematosus: correlation with treatment and disease activity. Lupus 21(9): 969977.

36. Luo XM, Edwards MR, Mu Q Yu Y, Vieson MD, et al. (2018) Gut Microbiota in Human Systemic Lupus Erythematosus and a Mouse Model of Lupus. Applied and environmental microbiology 84(4): 1-10. 